\title{
Correction to: The IICR and the non-stationary structured coalescent: towards demographic inference with arbitrary changes in population structure
}

\author{
Willy Rodríguez · Olivier Mazet · Simona Grusea - Armando Arredondo · Josué M. Corujo · Simon Boitard • \\ Lounès Chikhi
}

Published online: 17 February 2021

(c) The Author(s), under exclusive licence to The Genetics Society 2021

\section{Correction to: Heredity \\ https://doi.org/10.1038/s41437-018-0148-0}

The original version of this article unfortunately contained a mistake. In the article, the authors wrote that they used the mutation rate of $1.25 \mathrm{e}-8$ (which is the now generally accepted mutation rate for humans) but they actually had used the older mutation rate of $2.5 \mathrm{e}-8$, which they had also used in a previous article (Mazet et al, 2016, Heredity) for comparison with the article of $\mathrm{Li}$ and Durbin (2011) which used that older value. All calculations and figures were done with this older $2.5 \mathrm{e}-8$ mutation rate. This error only affects two locations in the manuscript (pages 673 and 674). The inferred values of the sizes of the human and Neanderthal demes should be replaced by 638 for humans and 100 for Neanderthals (instead of 1276 and 200, respectively) in page 673. It also affects a paragraph in page 674 where they mention the mutation rates.

More precisely, the authors propose the following two changes in the text:

\section{(1)}

Current sentence: Assuming a mutation rate per generation equal to $1.25 \mathrm{e}-8$, the proposed scenario is consistent with a deme size of 1276 for humans and a deme size of 200 for Neanderthals.

Corrected sentence: Assuming a mutation rate per generation equal to $2.5 \mathrm{e}-8$, the proposed scenario is consistent with a deme size of 638 for humans and a deme size of 100 for Neanderthals.

(2)

Current sentence: "In Mazet et al. (2016) and Chikhi et al. (2018) we used the mutation rates of $\mathrm{Li}$ and Durbin (2011) but here we used the values of Prufer et al. (2014) to be able to compare our IICR results to the PSMC results obtained by the latter study."

Corrected sentence: "As in Mazet et al. (2016) and Chikhi et al. (2018) we used the mutation rates of $\mathrm{Li}$ and Durbin (2011)." 